\title{
Is fatty liver indicative of a risk of metabolic syndrome among non-obese subjects?
}

\author{
Makoto Daimon ${ }^{1}$
}

Received: 29 June 2015/Published online: 21 July 2015

(C) The Japan Diabetes Society 2015

Metabolic syndrome (MetS), a complex of interrelated risk factors for cardiovascular disease (CVD), includes elevated fasting plasma glucose (FPG) levels, elevated blood pressure (BP), elevated triglyceride (TG) levels, reduced highdensity lipoprotein cholesterol (HDL-C) levels, and obesity [1]. Obesity seems to be a central metabolic risk factor (MetRF) because insulin resistance, which is generally related to obesity, is a potential pathophysiologic link among MetRF. In this context, obesity, as measured by abdominal adiposity and increased waist circumference, is included as an essential factor for diagnosis of MetS by the International Diabetes Federation (IDF) and the Japanese Examination Committee for Criteria of Metabolic Syndrome [2, 3]. However MetRF were frequently clustered, even among non-obese subjects. Subjects with clustering of MetRF are often insulin-resistant compared with subjects without clustering of MetRF, even though they were not obese. Thus, non-obese subjects with a clustering of MetRF are at increased risk of CVD, as are obese subjects with a clustering of MetRF, or subjects with MetS. Thus, evaluation of MetRF seems to be important for finding such individuals.

Recently, Hiraoka et al. reported an association of fatty liver with MetRF and such insulin-resistance indices as HOMA-R among non-obese subjects with normal waist circumference none of whom met the MetS criteria [4]. Blood pressure, serum triglyceride, and HOMA-R levels were significantly elevated among non-obese subjects with

Makoto Daimon

mdaimon@hirosaki-u.ac.jp

1 Department of Endocrinology and Metabolism, Hirosaki University Graduate School of Medicine, Hirosaki, Aomori 036-8562, Japan normal waist circumference and fatty liver compared to those without (males: $122 / 78$ vs. $120 / 76 \mathrm{mmHg}, 161.5$ vs. $105.5 \mathrm{mg} / \mathrm{dl}, 1.9$ vs. 1.3 , respectively; females: $120 / 73$ vs. $116 / 71 \mathrm{mmHg}, 115.2$ vs. $82.0 \mathrm{mg} / \mathrm{dl}, 1.7$ vs. 1.3 , respectively). This result indicated that those with fatty liver were at risk of clustering of MetRF and, thus, of developing MetS, even though they were not obese or did not meet the MetS criteria. Therefore, evaluation of fatty liver, which can be detected easily by ultrasonography in ordinary clinical settings, seems to be important for non-obese and obese individuals.

Furthermore, because the authors excluded those who regularly drank $20 \mathrm{~g}$ or more alcohol per day from their study population, the fatty liver described in the report seems to be non-alcoholic fatty liver disease (NAFLD) [4]. Studies of MetS have revealed a J-shaped relationship between alcohol intake and the risk of MetS [5-7]. Indeed, light alcohol consumption was actually associated with a lower risk of MetS [6-8] whereas very heavy alcohol consumption was associated with a substantial increase in the risk of MetS [9]. However, the different relationships between alcohol intake and the risk of clustering of MetRF might be related to the different obesity of the subjects, i.e. non-obese versus obese. We recently reported that alcohol intake by non-obese males with a clustering of MetRF was significantly higher than by those without, but such differnce was not observed in obese males [10]. Furthermore, analysis of non-obese males stratified into the four groups on the basis of alcohol consumption (none, light (1-139 g/ week), heavy (140-279 g/week), and very heavy ( $\geqq 280 \mathrm{~g} /$ week)) revealed a positive linear association between amount of alcohol intake and clustering of MetRF [10], i.e., increasing alcohol intake seemed to increase the risk of clustering of MetRF among non-obese Japanese males whereas non-obese individuals with no or light alcohol 
consumption seemed to be at less risk. However, in the report by Hiraoka et al., even such individuals who were not obese, and who did not drink or were light drinkers, were shown to be more at risk if they had fatty liver [4].

Fatty liver must also be evaluated precisely for nonobese individuals with normal waist circumference and who do not drink or who drink lightly, because it is associated with MetRF and insulin resistance. Inclusion of fatty liver among the criteria for MetS seems to be a challenge in the future, and seems to be warranted by the report by Hiraoka et al. [4].

\section{Compliance with ethical standards}

Conflict of interest The author has no conflict of interest.

Human rights statement and informed consent This article does not contain any studies with human or animal subjects performed by the any of the authors.

\section{References}

1. Alberti KG, Eckel RH, Grundy SM, et al. Harmonizing the metabolic syndrome: a joint interim statement of the International Diabetes Federation Task Force on Epidemiology and Prevention; National Heart, Lung, and Blood Institute; American Heart Association; World Heart Federation; International Atherosclerosis
Society; and International Association for the study of obesity. Circulation. 2009;120:1640-5.

2. The Examination Committee for Criteria of Metabolic Syndrome. Definition and criteria of metabolic syndrome. J Jpn Soc Int Med. 2005;94:794-809.

3. Alberti KG, Zimmet P, Shaw J. IDF epidemiology task force consensus group. the metabolic syndrome-a new worldwide definition. Lancet. 2005;366:1059-62.

4. Hiraoka A, Imai Y, Shinbata Y, et al. Clinical features of fatty liver in nonobese Japanese without regular alcohol intake. Diabetol Int. 2014;6:60-5.

5. Ayabe M, Kumahara H, Morimura K, et al. Very short bouts of non-exercise physical activity associated with metabolic syndrome under free-living conditions in Japanese female adults. Eur J Appl Physiol. 2012;112:3525-32.

6. Cai H, Huang J, Xu G, et al. Prevalence and determinants of metabolic syndrome among women in Chinese rural areas. PLoS One. 2012;7:e36936.

7. Freiberg MS, Cabral HJ, Heeren TC, et al. Alcohol consumption and the prevalence of the metabolic syndrome in the US: a crosssectional analysis of data from the Third National Health and Nutrition Examination Survey. Diabetes Care. 2004;27:2954-9.

8. Wakabayashi I. Cross-sectional relationship between alcohol consumption and prevalence of metabolic syndrome in Japanese men and women. J Atheroscler Thromb. 2010;17:695-704.

9. Djoussé L, Arnett DK, Eckfeldt JH, et al. Alcohol consumption and metabolic syndrome: does the type of beverage matter? Obes Res. 2004;12:1375-85.

10. Kaino W, Daimon M, Sasaki S, et al. Lower physical activity is a risk factor for a clustering of metabolic risk factors in non-obese and obese Japanese subjects: the Takahata study. Endocr J. 2013;60:617-28. 